\title{
La nature de la nation : le climat et les gens du
} Brésil (1780-1836)

Nature and the Nation: Climate and the People of Brazil (1780-1836)

\section{Lorelai Kury}

Traducteur : Martim Tavares et Patrice Bret

\section{revues.org}

Édition électronique

URL : http://ahrf.revues.org/12125

DOI : 10.4000/ahrf.12125

ISSN : $1952-403 X$
Éditeur :

Armand Colin, Société des études

robespierristes

Édition imprimée

Date de publication : 1 septembre 2011

Pagination : 129-152

ISBN : 978-2-200-92700-4

ISSN : 0003-4436

Référence électronique

Lorelai Kury, "La nature de la nation : le climat et les gens du Brésil (1780-1836) », Annales historiques de la Révolution française [En ligne], 365 | Juillet-septembre 2011, mis en ligne le 01 septembre 2014, consulté le 01 octobre 2016. URL : http://ahrf.revues.org/12125; DOI : 10.4000/ahrf.12125

Ce document est un fac-similé de l'édition imprimée.

Tous droits réservés 


\title{
LA NATURE DE LA NATION : LE CLIMAT ET LES GENS DU BRÉSIL (1780-1836)
}

\begin{abstract}
L'article aborde le débat sur le déterminisme climatique dans les textes de certains hommes de science brésiliens, qui publièrent pendant la période des Lumières jusqu'aux années qui ont suivi l'indépendance. On croyait vivement à cette époque que le climat déterminait le caractère de ses habitants. Ainsi, les aspects naturels du Brésil étaient des éléments essentiels pour la configuration même de l'identité. Les publications sur le climat du Brésil et sur les Brésiliens accompagnent la formation et l'activité d'une lignée de naturalistes, d'hommes de lettres et d'administrateurs, qui voyaient dans les Lumières une incitation aux réformes, une façon de jouir rationnellement du pouvoir des climats chauds, en évitant ses traquenards, mais en sachant exploiter ses presque infinies ressources.
\end{abstract}

Mots-clés : nature brésilienne, indépendance du Brésil, climat, José Bonifácio de Andrada e Silva, presse brésilienne, Lumières au Brésil

« Le gouvernement a beaucoup de pouvoir sur les plantes, il a tout pouvoir sur les hommes $»$. José Bonifácio de Andrada e Silva

L'une des caractéristiques les plus importantes de l'époque de l'Indépendance du Brésil (1822) est l'abondance de textes valorisant le pays et ses habitants. Cette thématique prit corps dans un débat assez vif sur la possibilité d'édifier une société civilisée dans une région tropicale, peuplée de métis, de blancs, de noirs et d'indigènes amérindiens. La seule situation géographique de l'Amérique portugaise était vue comme une 
entrave au développement des activités dites « de l'esprit ». Si cette opinion n'était pas nouvelle, elle a été la cible de débats scientifiques et philosophiques à partir du moment où des auteurs nés au Brésil se mirent à considérer comme centrale l'appartenance à l'espace public d'une patrie située sous les tropiques. En outre, l'esclavage des noirs et l'existence d'une population indigène non négligeable ont fait émerger des réflexions sur l'hétérogénéité physique et culturelle des habitants d'outremer.

Cet article analyse la représentation des particularités du climat et des habitants du Brésil ${ }^{1}$ dans des textes scientifiques de la période comprise entre les Lumières et la première génération romantique. Les communautés politiques, scientifiques et littéraires étant alors à peine différenciées et la presse spécialisée n'existant pas encore, les journaux proposaient des informations et des débats sur des thèmes variés. Mais dès l'époque de la colonisation portugaise, la question de l'identité brésilienne fut décisive pour parvenir à l'indépendance politique ${ }^{2}$. Dans un système social fondé sur la croyance de l'action déterminante de l'environnement sur la conformation physique et morale des êtres humains, les discussions sur le climat du Brésil et sur le caractère de ses habitants, qui furent explicitement soulevées en diverses occasions, mettaient toujours en relation les aspects naturels et sociaux. La presse plaçait aussi la question cruciale de l'esclavage au Brésil au croisement de phénomènes de la nature et de la société.

\section{Les Lumières et la culture de l'imprimé}

La presse fut un moyen important pour la formation et la consolidation des communautés littéraires, politiques et scientifiques au Brésil. Elle apparut dans ce pays au moment même où survint le changement politique et symbolique de son statut. L'Imprimerie Royale de Rio de Janeiro fut créée en 1808, quand le Prince Régent ${ }^{3}$ et la Cour s'établirent dans cette ville. Quelques années plus tard, en 1815, l'érection du Brésil en une des composantes du Royaume-Uni du Brésil, du Portugal et de l'Algarve conduisit à un fort sursaut identitaire américain. La formation

(1) L'unification de tout le territoire date seulement de 1823, mais le mot «Brésil» sera utilisé quelquefois ici pour désigner toute l'Amérique portugaise, y compris l'état du Grand Para (Grão Pará e do Maranhão).

(2) Sur l'histoire de la presse et la formation de l'espace public au Brésil, voir Marco MoRel, As transformações dos espaços públicos : imprensa, atores politicos e sociabilidades na cidade imperial (1820-1840), São Paulo, Hucitec, 2005.

(3) Devenu João VI en 1816. 
de l'espace public des lettres et celle d'une identité politique brésilienne se firent simultanément.

À l'époque des Lumières, les hommes de lettres et de sciences lusoaméricains étaient intégrés à l'univers des institutions de la métropole, où étaient les sphères de légitimation et les possibilités de circulation imprimée. Ils se formaient en Europe, principalement à Coimbra, et publiaient de préférence dans les circuits éditoriaux du Portugal, en particulier dans les mémoires de l'Académie Royale de Sciences de Lisbonne, fondée en 1779.

Un certain nombre de Brésiliens jouèrent un rôle central dans la politique de modernisation du Portugal, initiée dès les années 1760 par le ministre Marquis de Pombal. Au tournant des XVIII et XIX ${ }^{\mathrm{e}}$ siècles, leur présence était visible dans diverses sphères, surtout autour du ministre D. Rodrigo de Sousa Coutinho et du naturaliste padouan Domenico Vandelli, directeur du Jardin Botanique de l'Ajuda à Lisbonne. L'une des principales initiatives de l'époque fut la création à Lisbonne, en 1799, de l'imprimerie de l'Arco do Cego, dirigée par un naturaliste brésilien, le Frère José Mariano da Conceição Veloso. Cette maison d'édition ${ }^{4}$ publia d'innombrables ouvrages relatifs aux pratiques éclairées, européennes et coloniales, concernant l'exploration de la nature et le développement des sciences et des arts. Le Portugal confirmait alors son désir d'adhérer à un nouveau modèle de production et de diffusion des connaissances, fondé sur la circulation des textes imprimés et des images ${ }^{5}$.

Jusqu'au milieu du XVIII ${ }^{\mathrm{e}}$ siècle, très peu fut publié sur l'Amérique portugaise car les autorités censuraient tout ce qui pouvait fournir aux puissances étrangères des renseignements sur les produits coloniaux. Un des exemples les plus saisissants de cette politique du secret qui guidait les autorités portugaises fut la destruction du livre Cultura e opulência do Brasil por suas drogas e minas (Culture et richesse du Brésil par ses drogues et ses mines) du jésuite Antonil (Giovanni Antonio Andreoni), publié en 1711, parce qu'il contenait des informations sur la localisation des richesses et les méthodes de préparation du sucre. En 1800, la politique en matière d'édition s'était transformée : Veloso publia à l'Arco do Cego un extrait du livre d'Antonil sous le titre Extracto sobre os engenhos de assucar do Brasil, e sobre o methodo já então praticado na factura

(4) Voir Fernanda Maria Guedes de Campos (dir.), A Casa Literária do Arco do Cego (17991801), Lisbonne, Imprensa Nacional-Casa da Moeda - Biblioteca Nacional, 1999.

(5) Voir Lorelai KURY, « Homens de ciência no Brasil : impérios coloniais e circulação de informações (1780-1810) », História, Ciências, Saúde-Manguinhos, 11, 2004, supl. 1, p. 109-129. 
deste sal essencial (Extrait sur les moulins à sucre du Brésil, et sur la méthode pratiquée dans la fabrication de ce sel essentiel).

Cet éditeur publia de nombreuses traductions d'œuvres françaises et anglaises, principalement sur l'histoire naturelle appliquée, ainsi que des manuels d'enseignement de mathématiques, de navigation, de chimie, de gravure, ou de la poésie. Une des marques particulières des livres publiés par Veloso était l'usage intensif et didactique des images. L'éditeur chercha à se doter des techniques les plus modernes disponibles à l'époque ${ }^{6}$. Ainsi, les Lumières et la culture imprimée allaient de pair au Portugal. Le transfert de la Cour vers Rio de Janeiro et l'installation de l'imprimerie au Brésil modifièrent de façon substantielle la possibilité même de concevoir des auteurs et un public locaux, identifiés par leur appartenance à la patrie, puis à la nation brésilienne. À la suite de la gazette Idade d'Ouro do Brasil (L'Âge d'or du Brésil, 1811-1823), de Bahia, et du jounal $O$ Patriota (Le Patriote), publié à Rio de Janeiro en 1813 et 1814, plusieurs revues de variétés et de nouvelles publièrent des articles scientifiques tout au long du XIX ${ }^{\mathrm{e}}$ siècle.

De caractère encyclopédique, $O$ Patriota fut le premier périodique à publier des articles scientifiques au Brésil. Il contient des dizaines de textes qui englobent les thèmes les plus divers, comme la médecine, l'agriculture, les voyages, l'histoire, la politique et la poésie. Ce mélange de domaines, typique de la culture de l'époque, démontre le poids que la science avait acquis dans la mouvance intellectuelle des Lumières tardives luso-brésiliennes. Son éditeur fut un professeur de mathématiques de la nouvelle Académie militaire, le Bahianais Manoel Ferreira de Araujo Guimarães, également responsable de la Gazette du Rio de Janeiro. Ce journal peut être considéré comme la continuation, au Brésil, de la politique éditoriale promue par Veloso à l'Arco do Cego, sous les auspices de D. Rodrigo de Sousa Coutinho. Les publications de Veloso ont déjà été décrites comme le succédané de l'Encyclopédie de Diderot et D'Alembert au Portugal. O Patriota hérita du caractère encyclopédique de la publication portugaise mais cette fois, c'est à partir du Brésil que rayonnait le savoir.

Les périodiques spécialisés dans les sciences naturelles ne sont apparus au Brésil que dans la seconde moitié du XIX ${ }^{\mathrm{e}}$ siècle, avec la publication des séances de la Sociedade Velosiana, en 1851, et les Archivos do

(6) Miguel Figueira de Faria, A imagem útil, Lisbonne, Universidade Autônoma de Lisboa, 
Museu Nacional en 1876. Dans le cas de la médecine, cela arriva bien plus tôt. Les associations de médecins et les adeptes de divers systèmes de soins diffusèrent un abondant matériau dans les revues et les journaux ${ }^{7}$. Mais les sujets scientifiques tenaient déjà une place notable dans la production imprimée de la première moitié du siècle, que ce soit dans les périodiques médicaux ou les articles d'agriculture, dans les descriptions de voyages ou même dans la fiction ${ }^{8}$. Ce qui revenait le plus souvent était sans doute l'histoire naturelle « appliquée », comme on disait à l'époque, avec l'omniprésence de l'agriculture, notamment pour les questions relatives à la canne-à-sucre et plus tard au café. Ce domaine ne se manifestait pas seulement dans les organes consacrés à «l'industrie» (essentiellement agricole), comme O Auxiliador da Industria Nacional (L'Auxiliaire de l'industrie nationale), fondé en 1833, mais aussi dans des périodiques généralistes, comme $O$ Beija-Flor (Le Colibri, 1830), qui entendaient constituer une alternative aux journaux à caractère strictement politique.

En dehors des journaux, l'Imprimerie Royale de Rio joua un rôle décisif dans la diffusion des thèmes scientifiques au Brésil. Dans les premières décennies de son existence, elle publia des dizaines de traductions d'œuvres étrangères, concernant surtout la médecine et la chirurgie, ainsi que des manuels de mathématiques. En plus, beaucoup d'ouvrages d'utilité scientifique, écrits par des auteurs portugais et luso-américains, virent aussi le jour dans cette période. Ces titres servaient principalement de support à l'enseignement supérieur local, militaire et civil' .

Les sujets liés au climat et à la constitution physique des habitants de l'ancienne Amérique portugaise traversent l'édition brésilienne consacrée aux sciences. La question climatique n'intéressait pourtant pas seulement les naturalistes ou les médecins. Elle figurait au cœur des reconfigurations politiques et identitaires brésiliennes.

\section{L'acclimatation des lumières}

En 1836, pendant la période de la Régence qui succéda au règne de l'Empereur D. Pedro I ${ }^{\mathrm{er}}$, des Brésiliens publièrent à Paris deux numé-

(7) Cf. Luiz Otávio Ferreira, «Negócio, política, ciência e vice-versa : uma história institucional do jornalismo médico brasileiro entre 1827 e 1843 », História, Ciências, SaúdeManguinhos, Rio de Janeiro, 11, 2004, supl. 1, p. 93-107.

(8) Cf. Flora Sussekind, O Brasil não é longe daqui : o narrador, a viagem, São Paulo, Companhia das Letras, 1990.

(9) Pour une vision d'ensemble sur la presse et les institutions luso-brésiliennes, voir Maria Beatriz Nizza da SiLva, A cultura luso-brasileira, Lisbonne, Editorial Estampa, 1999. 
ros d'une revue appelée Nitheroy, qui allait devenir l'un des jalons symboliques du Romantisme littéraire brésilien. Les responsables en furent trois figures importantes de l'élite brésilienne cultivée : Manuel de Araújo Porto-Alegre, Francisco Salles Torres Homem et le poète Domingos José Gonçalves de Magalhães, un adepte de l'éclectisme philosophique de Victor Cousin. Magalhães publia dans la revue un "Essai sur l'histoire de la littérature au Brésil », dans lequel il exprimait quelques présupposés servant de base à ses réflexions sur l'identité nationale brésilienne. Il abordait, comme un fait établi, le thème essentiel de l'influence du climat sur les individus et, sans développer ses arguments, renvoyait le lecteur à leur autorité :

« Cette réalité que la disposition et le caractère d'un pays exerce une grande influence sur le physique et le moral de ses habitants est tellement connue aujourd'hui que nous l'érigeons en principe et nous croyons inutile d'insister à la démontrer avec des arguments et des faits, présentés par tant de naturalistes et de philosophes. Buffon et Montesquieu l'ont assez démontré $»^{10}$.

Selon l'auteur, le climat et la nature du Brésil auraient naturellement influencé la sensibilité de ses premiers habitants. À une nature magnifique correspondrait un certain type de sensibilité particulièrement épurée. Il affirmait ainsi que les indigènes brésiliens étaient extrêmement musicaux :

«Ce Brésil béni par tant d'heureuses dispositions d'une nature abondante, devait nécessairement inspirer ses premiers habitants; les Brésiliens devaient naître musiciens et poètes. Et ceux qui en doutèrent continueront-ils à le faire? Quelques écrits anciens nous enseignent que certaines tribus indigènes se distinguaient par leur talent en musique et poésie, les plus talentueux entre tous étaient les Tamoios qui habitaient Rio de Janeiro $»^{11}$.

Quant aux nouveaux habitants du pays, leurs productions intellectuelles s'harmonisaient en quelque sorte avec la nature par un processus d'ajustement des racines européennes à la nature américaine : « La poésie brésilienne n'est pas une indigène civilisée; elle est une Grecque

(10) Domingos José Gonçalves de Magalhães, « Ensaio sobre a historia da litteratura do Brasil. Estudo preliminar », Nitheroy. Revista Brasiliense, t. 1, 11836, p. 132-159, cit. p. 153.

(11) Ibid., p. 155. 
habillée à la française et à la portugaise et acclimatée au Brésil $»^{12}$. Ainsi, Gonçalves de Magalhães acceptait le présupposé des traditionnelles théories du climat mais il en inversait la polarité. De fait, la plupart de ceux qui ont écrit sur la nature brésilienne et de la musicalité des tropiques, affirmait exactement l'opposé, y compris Buffon. Le célèbre naturaliste fut un des principaux critiques de la nature américaine. Entre autres, il essaya, par exemple, d'expliquer ce qu'il pensait être une insuffisance mélodique des oiseaux d'Amérique : le climat aurait une influence néfaste sur ceuxci, qui, en outre, avaient pour unique modèle la voix des indigènes, que lui jugeait désagréable ${ }^{13}$.

Déjà loin de l'apogée des Lumières, le poète brésilien avait pour références les classiques du siècle antérieur. Les croyances traditionnelles furent également rénovées par d'autres auteurs qui s'interrogeaient sur le Nouveau Monde. Le philosophe Hegel fut l'un de ceux qui jugeaient négativement les sonorités du Nouveau Monde. Se fondant sur les rapports scientifiques des voyageurs Johann Baptist von Spix et Carl Friedrich Philipp von Martius, le professeur d'Iéna opposait la chaleur et le son :

« Les oiseaux tropicaux portent la chaleur qui ne préserve pas en ellemême, mais fonde et pousse vers la brillance métallique de la couleur cet être tel qu'en lui-même, cet état idéal intérieur comme voix ; cela veut dire que le son sombre dans la chaleur. La voix est en réalité quelque chose déjà de plus fort que le son, mais la voix se montre aussi dans cette opposition à la chaleur du climat $»^{14}$.

Ainsi, hommes, oiseaux et chaleur s'articulaient organiquement en ce lieu essentiellement tropical, hors de l'histoire et du progrès de $1^{\prime}$ esprit ${ }^{15}$. Le philosophe se référait à un extrait du récit de voyage de Spix et Martius au Brésil, réalisé entre 1817 et 1820, dans lequel les voyageurs décrivent le beau chant d'un oiseau brésilien. Ils affirmaient qu'il peut y avoir une certaine mélodie dans le chant des oiseaux brésiliens,

(12) Ibid., p. 146.

(13) Antonello Gerbi, La Disputa del Nuovo Mondo. Storia di una polemica (1750-1900), Milan, Adelphi Milano, 2000 [1955].

(14) HeGEL, article « O calor », § 303, Enciclopédia das ciências filosóficas em compêndio : 1830, São Paulo, Loyola, 1997, vol. II (A Filosofia da Natureza), p. 197. Édition française par Bernard Bourgeois, Paris, Vrin, 2004.

(15) Outre les diverses références extraites de l'œuvre de Spix et Martius, d'autres relations de voyageurs au Brésil seraient des sources importantes pour Hegel à propos du « retard » culturel des peuples indigènes des tropiques comme produit des conditions « adverses » du milieu géographique et biologique, entre autres celles de Maximiliano de Wied-Neuwied et Henry Koster. 
bien qu'en général on affirme le contraire. Ils ajoutaient toutefois : «De toute façon on peut imaginer que, si un jour l'écho de sons quasi inarticulés d'hommes dégénérés [les indigènes] disparaît des forêts brésiliennes, beaucoup de chanteurs à plumes produiront de savantes mélodies $»^{16}$. Spix et Martius suggéraient ainsi que la voix des animaux serait une sorte d'imitation de la voix humaine. Rien de plus éloigné de la musicalité attribuée aux Tamoios par Gonçalves de Magalhães que les grognements indigènes prétendument observés par les voyageurs. Pourtant, malgré les jugements opposés, il y avait bien une concordance : le climat règne sur l'art, la beauté, le caractère, les coutumes et les aptitudes des hommes et des animaux.

De l'époque des Lumières à l'Empire du Brésil, le jugement sur le climat du pays et sur sa population constitua une prise de position politique. La question des Indiens est très complexe et englobe des thèmes tels que leur utilisation comme main-d'œuvre, en remplacement du travail servile des Africains. Le versant scientifique du sujet renvoie aux croyances sur le déterminisme climatique, élément essentiel pour la philosophie des Lumières, très peu mis en évidence par l'historiographie ${ }^{17}$. Le poète romantique Gonçalves de Magalhães attirait l'attention sur le fait que les indigènes étaient les peuples natifs de la terre ou, du moins, les mieux adaptés au climat du Brésil. Même le voyageur Martius devait tracer, en 1824, un profil moral des indiens d'Amazonie organiquement lié à la forêt :

« Sombre comme l'enfer, enchevêtrée comme le chaos, voici une forêt impénétrable de troncs gigantesques, depuis le delta de l'Amazone jusque bien au-delà du territoire portugais en direction de l'Ouest. [...] Il n'est pas étonnant que l'âme de l'indien, errante dans cet environnement, devienne sombre et que, poursuivie par les ombres de la solitude, elle pense voir partout des créations fantasmagoriques de son imagination frustre $»^{18}$.

Le thème de la présence de noirs africains et de mulâtres au Brésil est également complexe et central pour la compréhension de la période.

(16) Passages cités par Hegel, op. cit. La première édition du premier volume du Reise in Brasilien est de 1823. Voir l'édition brésilienne : J. B. von Spix e C. F. Ph. Von Martius, Belo Horizonte/São Paulo, Itatiaia/Edusp, Viagem pelo Brasil. 1817-1820, 1981, p. 115, vol.1.

(17) Voir David ARnold, The Problem of Nature, Environment, Culture and European Expansion, Cambridge, Blackwell, 1996 et Pierre Bourdieu, «Le Nord et le Midi : Contribution à une analyse de l'effet Montesquieu », Actes de la recherche en sciences sociales, t. 35, 1980, L'identité. p. 21-25.

(18) Carl F. Ph. von Martius, «A Fisiononomia do Reino Vegetal no Brasil», Arquivos do Museu Paraense, vol. III, 1943, p. 239-271, p. 246. Trad. de E. Niemeyer et C. Stellfeld. (Der Physiognomie des Pflanzenreich in Brasilien, Rede zur oeffentl. Sitz. Akad. Wiss, München, 1824). 
Certains auteurs regardent le métissage comme un fait naturel, mais le maintien de l'esclavage jusqu'à la fin du XIX ${ }^{\mathrm{e}}$ siècle donne à la question raciale, pour les noirs, des caractères différents de la question indigène. Quoi qu'il en soit, la production éclairée au Brésil tendait à reconnaître l'unité du genre humain.

Le concept de « race » n'était pas encore utilisé comme une catégorie pré-établie pour l'analyse des populations. La démarcation entre les peuples se fondait plutôt sur les particularités de chacun concernant les coutumes, le régime alimentaire et le climat de chaque région. Dans le référentiel théorique néo-hippocratique du temps, les coutumes étaient ce que l'on appellerait de nos jours la culture avec l'addition des institutions civiles et politiques. La diète concernerait tout ce qui, tout ce qui, solide ou liquide, entre dans les corps, y compris le degré de pureté des substances et leur action dans le métabolisme humain - calmante, stimulante, nourrissante, toxique, purgative, caustique. Le troisième facteur, le climat, comprend tout l'environnement dans lequel une population s'insère : les saisons, le régime des vents, les températures, l'humidité, la topographie, le type de terrain, la flore et la faune.

Le « racial turn » au Brésil a suivi grosso modo la chronologie internationale, c'est-à-dire qu'il était déjà clairement défini autour de $1840^{19}$. La « race » ne devint un élément biologique donné et un caractère essentiel que dans la seconde moitié du XIX ${ }^{\mathrm{e}}$ siècle, lorsque l'esclavage - ou du moins le trafic intercontinental - fut en voie d'extinction. Cela ne signifie pas pour autant que tous les habitants de la planète étaient considérés comme égaux ni que les hommes de science de l'époque étaient unanimement adversaires de l'esclavage africain.

Ainsi, jusqu'aux premières décennies du XIX ${ }^{\mathrm{e}}$ siècle, les variétés humaines étaient considérées, de façon générale, comme découlant des conditions environnementales et géographiques. L'un des caractères les plus significatifs de la pensée scientifique des Lumières était la complexité accrue de la notion de climat et de circonstances, qui allaient se fondre lentement dans le concept de milieu ${ }^{20}$. Quelques traits des conceptions scientifiques d'hommes des Lumières tels que Alexandre Rodrigues Ferreira, Manoel Arruda da Câmara et José Bonifácio de Andrada e Silva exemplifient de façon paradigmatique cette atmosphère

(19) Mark Harrison, Climates and Constitutions. Health, race, environment and British imperialism in India, 1600-1850, Oxford, Oxford Univ. Press, 1999. Vrin, 1985.

(20) Georges CANGUILHEM, « Le vivant et son milieu », dans La connaissance de la vie, Paris, 
intellectuelle. Chacun d'eux mobilisait des arguments théoriques et des exemples variés, provenant de situations brésiliennes ou de cas relatés par des voyageurs et des fonctionnaires coloniaux.

Le naturaliste bahianais Alexandre Rodrigues Ferreira (17561815), élève de Domenico Vandelli, entreprit un long voyage au Mato Grosso et au Rio Negro, entre 1783 et 1792. Ses deux dessinateurs représentèrent de multiples aspects de ses expéditions, y compris la physionomie et les coutumes des populations indigènes. Bien que Ferreira ait rédigé des mémoires et des récits de ses excursions, ce matériel ne fut pas publié à l'époque. La raison pour laquelle les résultats restèrent inédits donne lieu à des débats historiographiques mais le cas n'est pas exceptionnel $^{21}$, et le mouvement des " voyages philosophiques » idéalisés par Vandelli a eu des succès inégaux. En tout état de cause, une certaine préparation technique et scientifique des voyageurs était nécessaire pour mener à bien leurs tâches.

Pendant son séjour au Brésil lors de l'expédition amazonienne, Ferreira eut l'occasion de recommander la guerre contre les Mura. Il croyait aussi utile l'envoi d'au moins mille cinq cents esclaves africains par an vers le Grão-Pará et le Mato Grosso ${ }^{22}$. Malgré cela, du point de vue de l'histoire naturelle ou de la description physique et morale des « variétés » de l'espèce humaine, il semble considérer la couleur des hommes comme un caractère circonstanciel. Sur les noirs africains, il écrivit peu et ce ne fut jamais un thème central de ses mémoires. Il est vrai que leur présence dans les régions qu'il parcourut était relativement faible. En revanche, les indigènes méritent des descriptions détaillées, tant dans des textes écrits pour un relevé physique et politique de la population de l'Amérique portugaise, que dans des réflexions plus théoriques sur l'espèce humaine.

Une ligne interprétative assez précise guidait ses considérations sur la nature de ce qu'il appelait les sauvages américains : la croyance dans la détermination de causes extérieures sur la conformation phy-

(21) Cf. Lorelai Kury, « A filosofia das viagens : Vandelli e a história natural », O Gabinete de Curiosidades de Domenico Vandelli, Rio de Janeiro, Dantes Editora, 2008; Ângela Domingues, « Para um melhor conhecimento dos domínios coloniais : a constituição de redes de informação no império português em finais do setecentos ", História, Ciências, Saúde-Manguinhos, 8, supl., 2001, p. 823-838 et Ronald Raminelli, Viagens Ultramarinas; monarcas, vassalos e governo a distância, São Paulo, Alameda Casa Editorial, 2008.

(22) Eduardo GALvão et Carlos Moreira NETo, « Introdução », dans Alexandre RodRigues Ferreira, Viagem filosófica pelas capitanias do Grão Pará, Rio Negro, Mato Grosso e Cuiabá. Memórias - Antropologia, s. 1., Conselho Federal de Cultura, 1974, p. 19. 
sique et morale des hommes. Le passage qui suit, extrait d'un mémoire sur des mammifères de la région amazonienne, dévoile ses conceptions :

« La diversité de leur couleur, les divers endroits où ils habitent, leurs mœurs et facultés corporelles, indiquent que, comme chez d'autres animaux, leur espèce aussi présente des variétés. Dans ce sens l'indien Tapuia en est une. Il est aussi homme que l'Européen, l'Asiatique ou l'Africain; en raison de la diversité de la couleur de sa peau et du pays où il habite, nous les appelons Tapuia selon le nom de leur propre langue [...] Les Tapuias n'ont pas d'autres différences que celles qui sont accidentelles chez tout être humain $»^{23}$.

À la différence de Ferreira, le Pernamboucain Manuel Arruda Da Câmara (1752-1810) publia divers mémoires de son vivant. Également lié aux groupes éclairés de l'entourage de Rodrigo de Sousa Coutinho, Câmara publia des textes dans les Mémoires de l'Académie des Sciences de Lisbonne, aux éditions de l'Arco do Cego et, à titre posthume, dans le journal $O$ Patriota. Ce médecin et naturaliste, propriétaire de terres et d'esclaves, se lança dans des projets de perfectionnement agricole et peut être considéré comme un "agriculteur éclairé ». Formé par Chaptal à Montpellier, où il obtint son doctorat de médecine en 1791, il rendit hommage à son maître en donnant son nom à une nouvelle espèce végétale (Chaptalia pekiy), qu'il mentionne dans son Paládio Português (Défenseur portugais, 1796) et cite à nouveau dans une note de son mémoire sur le cotonnier $^{24}$. Aussi le Brésilien prit-il pour modèle la chimie appliquée à l'agriculture développée en France.

Dans sa thèse de l'université de Montpellier en 1791, Câmara défend l'idée que la patrie naturelle des êtres humains est voisine des tropiques, où la température extérieure est égale à celle du corps humain, tandis qu'ailleurs les gens doivent déployer pour se vêtir une stratégie qui

(23) Alexandre Rodrigues FerReIRA, « Observações gerais e particulares sobre a classe dos mamíferos observados nos territórios dos três rios das Amazonas, Negro, e da Madeira : com descrições circunstanciadas, que quase todos eles, deram os antigos, e modernos naturalistas, e principalmente, com a dos Tapuios », dans Viagem filosófica pelas capitanias do Grão Pará, Rio Negro, Mato Grosso e Cuiabá. Memórias - Zoologia e botânica, s. 1., Conselho Federal de Cultura, 1972, p. 74.

(24) Manoel Arruda da CÂmara, Memória sobre a cultura dos algodoeiros, Lisbonne, Oficina Literária do Arco do Cego, 1799. Reproduzida em M. A. da CÂMARA, Obras Reunidas (dir. José Antonio Gonsalves de Mello), Recife, Fundação de Cultura da Cidade do Recife, 1982. 
n'est pas naturelle ${ }^{25}$. Dans un texte plus tardif, il joint à cette argumentation la facilité de s'alimenter dans la région intertropicale :

« Si nous jetons un coup d'œil philosophique sur la surface du globe, nous verrons que les pays situés entre les Tropiques paraissent être les seuls que la nature ait destinés à l'établissement de l'homme; car c'est seulement à cet endroit qu'il peut vivre commodément sans le secours de l'art et se nourrir à partir des innombrables fruits que la terre lui fournit en quantité prodigieuse, ce que l'on ne rencontre pas dans les pays avoisinant les pôles $»^{26}$.

Le Brésil devenait ainsi le lieu naturel de la vie. Câmara se positionnait pourtant à contre-courant de la littérature jusqu'alors dominante, avec des arguments très éloignés des portraits paradisiaques des chroniqueurs coloniaux. S'appuyant sur la chimie et l'histoire naturelle, apprises dans les grands centres européens et appliquées à son expérience de planteur, éleveur et maître d'esclaves de la capitainerie de Pernambouc, il cherchait à agir comme un anti-Buffon, prenant le contre-pied des idées du grand naturaliste français à propos d'une prétendue infériorité de la nature du Nouveau Monde et dégénérescence des humains vivant hors des climats tempérés.

Malgré leurs différences d'approche, Ferreira et Câmara cherchaient à traiter les caractéristiques de la région tropicale comme naturelles. L'historienne Maria Odila da Silva Dias a identifié leur génération comme la première qui chercha à adapter les connaissances européennes à la vie sur les terres brésiliennes. De cette expérience sont issues des solutions propres, originales et adaptées au milieu ${ }^{27}$. Je crois que, justement par suite de ce processus de maîtrise du Brésil, l'évaluation de l'influence du climat a été une des bases de réflexion de cette génération. Quelques décennies plus tard, lorsque les hommes de lettres et de sciences furent confrontés à la construction d'une identité nationale, la question fut plus amplement développée et ses contours se précisèrent avec José Bonifácio de Andrade e Silva (1763-1838) dont la mémoire fut respectée par les

(25) Manuel ARruda DA CÂMARA, Disquisitiones quaedam physiologico-chemicae, de influentia oxigenii in oeconomia animali..., Montpellier, Joannem Martel nato Majorem, 1791, dans Manuel ARRuda da CÂMARA, Obras reunidas, op. cit., p. 80-81.

(26) Id., Discurso sobre a utilidade da instituição de jardins nas principais províncias do Brasil, Rio de Janeiro, Impressão Regia, 1810, Ibid., p. 198.

(27) Maria Odila DA SILva Dias, «Aspectos da Ilustração no Brasil » (1968), dans $A$ interiorização da metrópole e outros estudos, São Paulo, Alameda, 2005. 
hommes de science du $\mathrm{XIX}^{\mathrm{e}}$ siècle, comme le zoologue Emílio Joaquim da Silva Maia, l'un de ses biographes, mais que les Brésiliens - qui ont coutume de l'appeler seulement par ses prénoms - considèrent surtout comme l'un des pères de la nation brésilienne.

Né à Santos, capitainerie de São Paulo, José Bonifácio séjourna une grande partie de sa vie en Europe. Au cours de son cursus à Coimbra, en 1790, Vandelli l'envoya en voyage d'études - surtout dans le domaine de la minéralogie - dans plusieurs pays européens. Plus tard, de 1812 à 1819, il fut secrétaire de l'Academia Real das Ciências de Lisboa. Il publia en français, en anglais et en allemand des mémoires dans d'importants périodiques scientifiques de l'époque ${ }^{28}$, tels le Journal de Physique, de Chimie, d'Histoire Naturelle et des Arts, édité par Delamétherie, ou le Allgemeines Journal der Chemie, de Leipzig. Il a écrit sur les diamants du Brésil et autres minéraux, sur la fabrication économique des chapeaux et sur les serpents à sonnette. Connu en France sous le nom d'Andrada, il y fut membre de la Société linnéenne, de la Société philomathique et de la Société d'histoire naturelle de Paris. En août 1790, il fit partie de la délégation de naturalistes qui présenta à l'Assemblée nationale une pétition en faveur de l'érection d'un buste de Linné29. Les liens unissant le Brésilien à la mémoire du naturaliste suédois furent d'ailleurs profonds, surtout en ce qui concerne les considérations sur «l'économie de la nature ». José Augusto Pádua a souligné la modernité de la pensée environnementale de José Bonifácio, liée à la tradition initiée par des administrateurs coloniaux - tel Pierre Poivre à l'île de France - qui consistait en une rationalisation de l'exploitation des forêts afin de préserver l'humidité et la fertilité nécessaires à l'agriculture et à l'élevage ${ }^{30}$.

$\mathrm{Au}$ sein de la sphère scientifique naissante dans laquelle évoluaient les Brésiliens, des deux côtés de l'Atlantique, le minéralogiste appartenait au groupe éclairé proche du ministre D. Rodrigo de Sousa Coutinho, mort en 1812. Il était notamment en correspondance avec Domingos Borges de Barros, l'un des principaux rédacteurs de O Patriota, dans lequel il

(28) Voir Obras cientificas, politicas e sociais de José Bonifácio de Andrada e Silva, Edgard de Cerqueira Falcão (éd.), s.l., s.n., 1963, 3 vol.

(29) Adresse des naturalistes à l'Assemblée nationale. Du 5 août 1790, Paris, Imprimerie nationale, 1790, 4 p. Voir Pascal DuRis, Linné et la France, Genève, Droz, 1993, p. 81-82, p. 95 et Jean-Luc Chappey, Des naturalistes en Révolution. Les procès-verbaux de la Société d'histoire naturelle de Paris (1790-1798), Paris, Éd. du CTHS, 2009, p. 21-22.

(30) José Augusto PÁduA, Um sopro de destruição. Pensamento politico e crítica ambiental no Brasil escravista (1786-1888), Rio de Janeiro, Jorge Zahar, 2002, chap. 3. Voir aussi Richard Grove, Green Imperialism, Cambridge, Cambridge University Press, 1995. 
publia un article de minéralogie. Enfin, à plus de cinquante ans, en 1819, il rentra au Brésil et commença à s'immiscer dans les débats politiques qui devaient conduire à l'Indépendance.

Les références au climat et à la spécificité des différentes régions brésiliennes et de ses habitants parsèment toute l'œuvre de José Bonifácio mais, jusqu'à son retour dans sa patrie, ses réflexions sont plus générales. Ses préoccupations portaient en grande partie sur l'administration rationnelle des sciences et des arts. Certes, le thème des particularités nationales était déjà présent dans ses écrits, mais surtout sous forme de comparaisons entre le Portugal et les autres pays européens dont le but était de souligner le besoin de réformes dans l'empire portugais. Sa condamnation de l'esclavage était claire, tant par rapport au traitement inhumain donné aux esclaves que par le manque d'économie et de rationalité qui en découlait. À partir de 1819, José Bonifácio trouva le Brésil dans une situation différente, car la présence du souverain à Rio de Janeiro avait déplacé le centre du pouvoir vers l'Amérique. Cette situation devint intenable après le mouvement connu sous le nom de «la Révolution de Porto », qui commença d'exiger le retour du roi au Portugal. Dès lors, l'énergie du savant brésilien se porta sur la spécificité du cas brésilien et, après l'Indépendance, il proposa même des mesures concrètes pour dépasser petit à petit l'esclavage et employer les indiens comme travailleurs libres.

Durant ces années, pendant lesquelles José Bonifácio exerça une prééminence politique et une influence sur Pedro, l'héritier du roi João VI, il condensa dans quelques écrits une série de propositions pour le développement de la civilisation au Brésil, lesquelles articulaient les conceptions et pratiques des Lumières et son expérience brésilienne. Mais à la fin de 1823, peu après l'Indépendance et l'accession de Pedro ${ }^{\text {er }}$ au trône impérial, il tomba en disgrâce et gagna la France, où il passa plus de cinq ans en exil à Bordeaux. Ses plans sur les indiens, l'esclavage et même sur diverses activités économiques ne devaient pas être mis en pratique.

Cette même année, José Bonifácio avait en effet présenté deux projets de loi à l'Assemblée générale constituante: Apontamentos para a Civilização dos Índios Bravos do Império do Brasil (Notes pour la civilisation des indiens sauvages de l'Empire du Brésil), publié aussitôt par l'Imprimerie nationale (1823), et Representação à Assembléia Geral Constituinte e Legislativa do Império do Brasil sobre a Escravatura (Représentation à l'Assemblée générale constituante et législative de l'Empire du Brésil sur l'Esclavage), publiée plus tard, en portugais par Firmin Didot à Paris en 1825, et en anglais par Butterworth à Londres en 1826. 
Dans le premier texte ${ }^{31}$, Andrada décrit les indiens comme des barbares paresseux et sans freins pour régler leurs passions, auxquels, toutefois, ne manquent ni le « feu naturel de la raison ${ }^{32}$ » ni la capacité de se perfectionner. Car, selon le naturaliste, «l'homme primitif n'est ni bon ni mauvais naturellement, il est juste un automate, dont les ressorts peuvent être actionnés par l'exemple, l'éducation et des avantages $»^{33}$. Mieux, il ajoutait même : "Si Newton était né parmi les Guaranis, il aurait été un bipède de plus, qui [aurait marché] à la surface de la terre; mais un Guarani élevé par Newton aurait peut-être pu prendre sa place $»^{34}$.

« L'indien sauvage », selon lui, n'a pas recours à la raison car la nature lui offre tout ce dont il a besoin, comme la chasse et la pêche abondantes, et les fruits de la forêt. Il ne connaît ni distinctions sociales ni ambitions. Comme il n'a rien à calculer, il méconnaît les « idées abstraites de la quantité et du nombre $»^{35}$. La nature brésilienne devient alors une composante de base pour la compréhension de la question indigène. Dans un manuscrit non daté, José Bonifácio explicite encore une fois son interprétation : «Dans les climats fertiles du Brésil, les indiens n'avaient aucune raison de former de grandes sociétés, parce que seuls les besoins, puis la coutume requièrent le regroupement $\|^{36}$.

Malgré des variantes individuelles, le traitement donné aux questions brésiliennes s'inscrivait donc dans une vision universaliste de la nature humaine mais aussi dans la croyance en un déterminisme du milieu naturel sur la société. Les groupes liés à Domenico Vandelli, au Frère Veloso et à D. Rodrigo de Sousa Coutinho ont établi une espèce de lignée qui voyait dans les Lumières une incitation aux réformes, une façon rationnelle de mettre à profit le pouvoir des climats chauds, évitant ses pièges, mais sachant en explorer les apports presque infinis.

(31) Sur les débats autour de la politique indigène de l'époque, voir John M. MonTEIRo, «Os planos de civilização e os índios », dans Ana Silvia Volp Scott et Eliane C. Deckmann Fleck, A Corte no Brasil : População e Sociedade no Brasil e em Portugal no início do século XIX, São Leopoldo, Oikos/Unisinos, 2008.

(32) José Bonifácio DE Andrada E SiLva, Apontamentos a para Civilisação dos Indios Bravos do Imperio do Brasil, Paris, Firmin Didot, 1825, p. 3. Reproduit en fac-similé dans Obras cientificas, politicas e sociais, op. cit. t. II.

(33) Ibid.

(34) Ibid. Il y a un doute sur mot entre crochets : «pisara » (« aurait marché ») ou " pesara » (« aurait pesé »).

(35) Ibid., p. 2.

(36) Id., Projetos para o Brasil, Organização de Miriam Dolhnikoff, São Paulo, Companhia das Letras, 2005, p. 149. 
Chez Andrada, la tradition des Lumières se matérialisa en des propositions globales pour tout le Brésil. La question de l'esclavage mérita aussi une approche qui privilégiait la centralité de la nature brésilienne et comprenait les différences humaines comme des contingences. Il croyait qu'il fallait encourager les mariages entre blancs et indiens, ainsi qu'entre indiens, noirs et mulâtres. Malgré la difficulté de son exécution, cet « amalgame »- pour utiliser une métaphore minéralogique présente dans son lexique - serait une opération nécessaire pour former une nation homogène. Pour lui, l'esclavage était le pire mal qui rongeait le Brésil et empêchait sa civilisation. La présence d'un grand nombre de travailleurs non incités à recourir à la raison faisait du travail une activité stupide. Beaucoup de tâches qui pouvaient être réalisées par des machines simples se trouvaient accomplies par la force brute des esclaves. Cette critique de l'incompatibilité entre le progrès des arts et le travail servile est une constante de certains écrits du $\mathrm{XIX}^{\mathrm{e}}$ siècle, parmi lesquels les premiers journaux imprimés au Brésil. Pour José Bonifácio,

«La nature a tout fait en notre faveur, nous par contre nous n'avons rien ou très peu fait pour la nature. Nos terres sont désertes, et les rares terres que nous ayons défrichées sont mal cultivées, parce qu'elles le sont par des bras indolents et contraints; par manque de travailleurs actifs et instruits, nos nombreuses mines sont inconnues ou mal gérées; nos précieuses forêts disparaissent, victimes du feu et de la hache destructrice de l'ignorance et de l'égoïsme $»^{37}$.

Tout au long des publications, lettres et annotations de Andrada, il apparaît qu'un des axes de son effort intellectuel pour comprendre et transformer le Brésil est la prise en compte de la dynamique existant entre nature et civilisation, entre les conditions effectives et les réformes possibles. Par exemple, dans des manuscrits épars, on peut lire la phrase suivante : «Les chaleurs continuelles et les superstitions religieuses sont des entraves constantes à l'industrie et à l'activité $»^{38}$. La gravité de la constatation est toutefois résolue par la conviction que la volonté politique peut agir puissamment sur la société humaine : «Que l'on invoque à souhait les influences physiques, les besoins du climat, etc. Le gouvernement a beaucoup de pouvoir sur les plantes, il a tout pouvoir sur les

(37) Id., Representação à Assemblea Geral Constituinte e Legislativa do Imperio do Brasil sobre a Escravatura, Paris, Firmin Didot, 1825, p. 38. Reproduit en fac-similé dans Obras... de José Bonifácio de Andrada e Silva, op.cit. (note 32).

(38) Id., Projetos para o Brasil, op. cit., p. 188. 
hommes $»^{39}$. Un autre exemple, qui contredit les affirmations négatives de la tradition du XVIII ${ }^{\mathrm{e}}$ siècle à propos des climats chauds : " Les climats ne font rien, les Perses, conquérants avec Cyrus, furent vaincus et défaits sous Xerxès $\gg{ }^{40}$.

Cette espèce de tension et le fait même que les appréciations sur le climat du Brésil soient récurrentes témoignent de sa centralité. Dans quelques réflexions transparaissent la recherche de solutions et une analyse plus profonde de la question. Dans un texte resté manuscrit, Andrada imaginait une distribution géographique des habitants du pays qui suivrait leurs aptitudes naturelles : «Au Brésil les montagnes des provinces chaudes et les provinces froides devraient être habitées et cultivées seulement par des blancs et des indiens; et les terres basses et chaudes par des noirs, des cabras et mulâtres fils de noirs $»^{41}$.

La gradation climatique des aptitudes paraît parfois aller au-delà des espoirs de réformes et de la rationalisation des comportements, comme dans le texte suivant :

« Et comment les terres de Bahia, plaine fertile et verdoyante dispensatrice de délices, et l'indolence qui amoindrit ces valeurs, pourraientelles produire des gens vaillants et actifs? Mais São Paulo, montagneux et rugueux en partie, tempère la rudesse, la brutalité des peuples entièrement montagnards et habitants de pays glacés; et est sans aucun doute le plus capable de grandes choses. Ici les montagnes se mélangent avec la campagne et les vergers délicieux; le froid bat la chaleur et donne aux corps cette énergie, que ne connaissent pas les peuples d'autres climats $»^{42}$.

Natif de Santos, dans la capitainerie de São Paulo, Andrada est l'un des fondateurs d'une identité régionale pauliste, qui allait se fortifier au $\mathrm{XIX}^{\mathrm{e}}$ siècle, exaltant le climat doux de la province et les racines indigènes de ses élites blanches.

\section{Inversion : le gouvernement sous les tropiques}

Avec l'installation du Prince Régent D. João à Rio de Janeiro, en 1808, la possibilité de conjuguer tropiques et civilisation fut plus que jamais débattue. Dès la fin du XVIII ${ }^{\mathrm{e}}$ siècle, une phase de valorisation des

(41) Ibid., p. 180-181. Les cabras peuvent être des métis de noirs ou d'indiens.

(42) Ibid., p. 193. 
aspects naturels, économiques et politiques locaux avait commencé à remplacer une période marquée par la diminution de la production des mines d'or et la recherche d'une diversification de la production coloniale, rendue possible par une conjoncture extérieure favorable. Parallèlement à l'accroissement des recherches sur les produits locaux, l'acclimatation d'animaux et de plantes, et sur les pratiques agricoles et extractives, apparurent de nouvelles occasions de constitution de savoirs locaux, fruits des investigations et des expériences effectuées sur place. En 1772, c'est à Rio de Janeiro que fut fondée, par exemple, l'Academia Científica (Académie scientifique). Dans cette société furent lus des travaux d'astronomie, de médecine et d'histoire naturelle appliquée. La création de l'Académie avait été encouragée par le Vice-roi, le Marquis de Lavradio, responsable de diverses réformes dans la ville. Dans une lettre de mars $1772 \mathrm{au}$ Marquis de Angeja, D. Luis de Almeida Portugal écrit : «J'ai décidé de constituer un groupe de médecins, chirurgiens, botanistes, pharmaciens et quelques curieux, tant de cette capitale que de l'intérieur de cette Capitainerie, formant avec eux une assemblée ou académie afin d'examiner toutes les choses que l'on puisse rencontrer dans ce continent appartenant aux trois règnes : végétal, animal et minéral. » Il souhaitait, avec cette académie, « faire en sorte que nous ne continuons pas à avoir honte d'être toujours instruits par des étrangers qui profitent de choses précieuses que nous possédons ${ }^{43}$. Le pronom « nous » de la lettre du Vice-roi signifie tous les Portugais, habitants du Royaume et de l'outremer. C'est dans ces espaces que les hommes nés au Brésil allaient agir et que l'on commença à valoriser l'expérience native comme un élément important.

Près de cinquante ans plus tard, en 1819, alors que João VI résidait à Rio de Janeiro, l'Imprimerie royale publia un texte anonyme intitulé Algumas reflexões sobre a História Natural do Brasil... (Quelques réflexions sur l'histoire naturelle au Brésil et l'établissement du Musée et Jardin botanique à la Cour de Rio..., suivi d'une Instruction pour les voyageurs et employés dans les colonies ${ }^{44}$. L'auteur y soutenait que

(43) Marquês do LaVradio, Cartas do Rio de Janeiro, Rio de Janeiro, Instituto Nacional do Livro, 1978, p. 97. Sur l'académie, voir, entre autres, Regina BELTRÃo MARQUES, « Escola de homens de ciências : a Academia Científica do Rio de Janeiro, 1772-1779 », Educar, 25, 2005, p. 39-57. Sur les académies antérieures de l'Amérique portugaise, voir Íris KANTOR, Esquecidos e renascidos. Historiografia Acadêmica Luso-Americana (1724-1759), São Paulo, Hucitec; Salvador : Centro de Estudos Baianos/UFBA, 2004.

(44) [José Feliciano Fernandes PInHEIro], Instrucção para os viajantes e empregados nas colonias... precedida de Algumas reflexões sobre a História Natural do Brasil, e estabelecimento do Museu e Jardim Botânico em a Corte do Rio de Janeiro, Rio de Janeiro, Impressão Régia, 1819. 
« [c'est] seulement de naturalistes dignes et judicieusement employés au Brésil toute leur vie durant que l'on pourra attendre une série d'observations savamment faites, comparées, et systématisées qui apportent toute la lumière sur la nature de ces contrées et nous apprennent les moyens de la convertir à notre profit $»^{45}$. À ce moment, les institutions d'histoire naturelle du Portugal avaient déjà perdu leur importance en faveur de Rio de Janeiro. Bénéficiant de l'installation du souverain au Brésil depuis dix ans, cette inversion des centres du savoir fut couronnée par la fondation du Musée Royal en 1818.

Ce texte témoigne, selon moi, du changement symbolique d'une géographie scientifique et institutionnelle qui liait le Portugal et le Brésil. Son auteur était probablement José Feliciano Pinheiro (1774-1847), né à Santos comme d'Andrada, dont il fut un proche, plus tard Vicomte de São Leopoldo et l'un des fondateurs de l'Empire du Brésil. Avec la mort de Domenico Vandelli, en 1816, s'achevait aussi tout un cycle d'activités des jardins et collections du Portugal vers lequel convergeait un d'un réseau de voyageurs et correspondants coloniaux formés sous l'autorité du directeur de l'Ajuda. L'auteur des Algumas reflexões... établit un plan pour l'organisation du Musée Royal qui prévoyait que chaque Capitainerie posséderait un cabinet d'histoire naturelle, contenant tous les produits de la circonscription. La collecte devrait être faite en deux exemplaires, afin qu'une collection fût envoyée au musée central, à Rio de Janeiro. Les îles et possessions africaines et asiatiques devaient suivre le même modèle l'Indépendance n'advint que trois ans plus tard. L'auteur appelait ainsi à la constitution d'un réseau ayant pour centre la nouvelle capitale et se substituant à l'organisation maintenue par Vandelli pendant des décennies, avec Lisbonne pour épicentre.

Non seulement ce texte fixait les paramètres de la consolidation des activités scientifiques au Brésil, mais, au-delà de l'inversion des rapports entre Lisbonne et Rio de Janeiro, il faisait aussi des questions de la biogéographie de l'époque l'un des thèmes centraux. N'intéressant pas le seul domaine des spécialistes de l'histoire naturelle, les Algumas Reflexões... traitaient du débat sur la spécificité de la nature au Brésil, revendiquant pour les Brésiliens le rôle de producteurs et gestionnaires du savoir. Il en ressort aussi que la définition même de la «nature brésilienne» se construisit lentement en liaison avec l'invention de l'unité naturelle et politique du territoire. Pour démontrer l'importance de l'établissement 
d'un musée au Brésil, l'auteur citait Buffon en ignorant les autres affirmations du naturaliste français à propos de l'infériorité de la faune américaine :

« Le grand Buffon l'ayant déjà annoncé, il est démontré aujourd'hui parmi les naturalistes, que, en dépit de quelque analogie de forme, tous les animaux qui n'ont pas les moyens de traverser l'Océan sont, dans la partie méridionale de l'Amérique, d'espèces différentes - et jusqu'à des familles entières - des animaux des autres parties de la terre, y compris ceux de l'Amérique septentrionale; il est clair que c'est seulement depuis cette partie méridionale que les musées du monde peuvent être approvisionnés en animaux qui n'existent que dans cette région $»^{46}$.

Pour la géographie des animaux, thème récurrent dans tout le texte, l'auteur ne citait pas de travaux plus récents que ceux de Buffon. Pour la botanique, il conseillait la lecture de l'Essai sur la géographie des plantes, de Humboldt. Enfin, dans son bilan de l'histoire naturelle internationale, portugaise et brésilienne, il signalait les œuvres essentielles produites au Brésil ou par des Brésiliens, comme le poème Caramuru de Santa Rita Durão (1781), la Corografia Brasílica de Aires de Casal (1817), le journal $O$ Patriota et les travaux de Frère Veloso, parmi d'autres œuvres moins connues, parfois manuscrites.

Ainsi, la situation géographique du Brésil présida à la fondation de la science brésilienne, symboliquement établie en 1819. Loin d'être mineur, cet aspect du problème se retrouve dans les discussions politiques à propos du retour de João VI. Dans ce que l'on appelle les « pamphlets de l'Indépendance », parus entre 1820 et 1822, une des questions les plus débattues était justement la possibilité pour une région "torride » et habitée par une population non blanche, comme le Brésil, d'accueillir un quelconque type de civilisation, voire de devenir le centre politique de l'Empire ${ }^{47}$.

Une des premières mesures de D. João en arrivant à Rio de Janeiro en 1808 avait été de commander au premier médecin du Royaume (Físico-Mor do Reino), Manuel Vieira da Silva, une expertise générale

(46) Ibid., p. IV.

(47) Voir Lúcia Maria Bastos P. das Neves, «A "guerra de penas" : os impressos políticos e a independência do Brasil », Tempo, t.4, n. 8, août 1999, p. 41-65; José Murilo de CARvalho, «O motivo edênico no imaginário social brasileiro ", Revista Brasileira de Ciências Sociais (online), t. 13, n. 38, automne 1998, et $O$ Debate político no processo da Independência (Introduction de Raymundo FAORO), Rio de Janeiro, Conselho Federal de Cultura, 1973. 
sur les mesures à prendre pour que la ville devienne moins insalubre. Les diagnostics existants sur la capitale brésilienne étaient les pires possibles. L'ancien Vice-Roi de Brésil, António Álvares da Cunha, en arrivant à Rio en 1763, rejeta l'idée de se fixer dans la résidence des gouverneurs, au Largo do Carmo, précisément là où le Prince Régent s'installa plus tard avec sa famille. Comme, traditionnellement, les collines étaient tenues pour plus salubres que les plaines, la plupart des ordres religieux s'y étaient installés. Aussi Cunha avait-il préféré résider au Morro do Castelo, dans l'ancien collège des Jésuites, pour fuir les moustiques et les échoppes des noirs ${ }^{48}$.

Un médecin éclairé, José Pinto de Azeredo, docteur en médecine de l'Université de Leyde, qui avait également étudié à Edimbourg, chercha justement à vérifier cette conviction enracinée de la supériorité de l'air des collines. Il publia en 1790, dans le Jornal Enciclopédico de Lisbonne, ses intéressantes expériences sur l'air de Rio de Janeiro, sa ville natale ${ }^{49}$.

Malgré la modernité de ses méthodes, Azeredo finit par corroborer le jugement habituel d'un climat tropical mauvais par nature. De ses expériences, il déduisit que les collines n'étaient pas plus salubres que les plaines et que « notre atmosphère contient généralement moins d'air pur et moins de fixe, mais plus d'air mophète qu'en Europe ». Or, selon lui, l'《 air mophète » (azote) aurait la capacité de « tuer passivement » car il ne pourrait pas attirer « les parties phlogistiques du sang » qui « nous sont nocives et mortelles ». Cette combinaison de peu « d'air fixe » (gaz carbonique), « destructeur de certaines causes nocives », et de beaucoup d'air mophète serait pernicieuse. Son hypothèse était que ce dernier « en attirant les mauvaises vapeurs des lagunes et les eaux stagnantes, en servant encore d'alimentation à certaines espèces d'insectes qui nous attaquent, [était] la cause des maladies. "

En 1808, le diagnostic de Manuel Vieira da Silva était tout autre. Bien qu'il fût d'accord avec d'autres médecins de l'époque sur plusieurs points de l'analyse, le premier médecin du Royaume entendait utiliser les connaissances disponibles pour agir de façon pragmatique et contourner les contraintes du climat tropical et de l'emplacement de la ville. Ses premières considérations étaient très semblables à celles de l'époque : une description de la plaine située entre collines, dans laquelle Rio de

(48) Nireu Cavalcanti, O Rio de Janeiro setecentista, Rio de Janeiro, Jorge Zahar, 2004, p. 37.

(49) José Pinto De Azeredo, « Exame quimico da atmosphera do Rio de Janeiro », Jornal Encyclopédico, Lisbonne, mars 1790, p. 259-288. 
Janeiro s'est établie. Son analyse présupposait une vision d'ensemble, celle, probablement, de quelqu'un qui s'est penché sur le plan de la ville. Le médecin avait besoin d'observer le tracé des rues, la direction des vents, l'emplacement des collines, les sources d'approvisionnement de la ville, les aliments consommés par les habitants, leurs habitudes. La médecine des Lumières est fondamentalement néo-hippocratique ${ }^{50}$, c'està-dire que trois variables entrent en ligne de compte pour un diagnostic de la santé de la population : l'environnement, les aliments, les habitudes.

Afin d'améliorer la santé de la population, le premier médecin recommandait que les efforts fussent dirigés vers l'extinction de ces causes qui, «étant l'œuvre des hommes, peuvent être anéanties par la même main qui les a fait naître ». Ainsi, il énumère : des marais sans écoulement; des sépultures dans les églises; le manque de lazarets pour que les «noirs » nouveaux venus y soient placés en quarantaine; le manque de réglementation sur les denrées alimentaires vendues dans la ville; des soins au bétail, depuis les pâturages jusqu'au commerce de la viande; le manque de bons médicaments. Il divergeait de la plupart des médecins de l'époque sur une question clé : l'arasement des collines. Beaucoup proposaient de détruire en particulier le Morro do Castelo afin d'améliorer la ventilation et de balayer les miasmes résultant des eaux stagnantes. Vieira da Silva, quant à lui, prônait le boisement des collines pour absorber les exhalations insalubres et renouveler l'air. Les études de l'époque se rapportant à la respiration des végétaux traçaient de nouveaux contours pour la compréhension de ce que l'on appelait «l'économie de la nature », expression d'ailleurs utilisée par le médecin pour démontrer les bénéfices des collines boisées.

De cette façon, la chaleur n'était pas traitée comme une barrière infranchissable : il était possible de lutter contre les maux inhérents à la situation climatique locale par des mesures de «santé publique » et des prescriptions individuelles. Cela ne veut pas dire qu'il n'y avait pas de critiques du climat tropical. Au contraire, notamment dans les textes antérieurs à 1808 , la chaleur et l'humidité de la ville sont exécrées. Un médecin portugais du nom de Medeiros a même affirmé que Rio était inhabitable

(50) Cf. Lorelai KurY, « Rio de Janeiro : a cidade e os médicos no período joanino », dans Ana Silvia V. Scott et Eliane C. D. Fleck (dir.), A Corte no Brasil... op. cit.; Henrique Cairus et Wilson Ribeiro Junior, Textos hipocráticos. O doente, o médico e a doença, Rio de Janeiro, Editora Fiocruz, 2005; Ludmila JoRdANOVA, « Earth Sciences and environmental Medicine : the synthesis of the late Enlightenment », dans Ludmila Jordanova et Roy PORTER (dir.), Images of the Earth. Essays in the History of the Environmental Sciences, Chalfont St. Giles, B.S.H.S., 1979. 
à cause de l'air pestientiel, chaud et humide que respiraient ses habitants. Rédigé en 1798, son texte fut publié dans le journal O Patriota, en 1813 et mérita une note de l'éditeur pour tenter d'adoucir le diagnostic :

« Nous devons dire dans l'intérêt de la vérité, qu'une grande partie des causes, tant physiques que morales, que ce médecin et d'autres ont désignées comme origine des maladies de Rio de Janeiro, ont disparu depuis que cette ville a l'honneur d'être la Cour de Notre Auguste Souverain, et tout particulièrement les causes morales; et si nous insérons ces trois avis dans ce Périodique, nous le faisons pour démontrer la sagesse des mesures que l'on a appliquées et augmenter l'espoir qu'elles puissent corriger les erreurs d'une situation morbide $\aleph^{51}$.

Les Lumières mettent non seulement en évidence l'usage de la raison par la «Philosophie » mais elles se distinguent aussi par la tentative de rationaliser la vie en société dans ses aspects les plus concrets et essentiels, en faisant du climat, des vents ou des immondices des objets de son action. Avec son instinct réformateur, José Bonifácio de Andrada e Silva croyait aussi qu'il était possible de s'adapter au climat local. Dans une lettre au Comte de Funchal, qui devait peut-être vivre dans la Cour tropicale, il affirmait :

« N'ayez pourtant crainte pour votre santé; car malgré la Nouvelle Guinée du Rio de Janeiro, si l'on suit la diététique [mot illisible] fondée sur une longue expérience, et si l'on ne travaille pas aux heures les plus chaudes, je suis sûr que vous vivrez aussi bien que les indigènes - les bains de mer et les promenades à cheval vous feront beaucoup de bien; et Dieu vous viendra en aide pour le reste $\aleph^{52}$.

L'un des visages des Lumières au Brésil pendant la période qui précède l'Indépendance se montre dans une lignée de naturalistes et d'hommes de science qui cherchèrent à réfléchir et à agir sur la spécificité brésilienne. Ils ont souvent choisi de rendre publiques leurs expériences

(51) [Manuel Ferreira de AraúJo Guimarães], O Patriota, mars 1813, p. 11, note a.

(52) Lettre de José Bonifácio de Andrada e Silva au Comte de Funchal, 30 juillet 1812, ms., Instituto Histórico e Geográfico Brasileiro. Transcription dans José Bonifácio DE ANDRADA E SiLVA, Projetos para o Brasil, op. cit., p. 169. 
par la voie de l'imprimé, soit au Portugal, soit dans la presse brésilienne naissante. La création d'espaces d'activité scientifique accompagna la formation même de groupes savants qui s'identifiaient à la patrie brésilienne, puis à la nation.

La perspective universaliste des Lumières se concrétisa fréquemment par des actions pratiques destinées à résoudre des problèmes quotidiens de façon méthodique et scientifique. Pour la génération qui fit l'Indépendance, leurs réflexions pour mettre en pratique la civilisation de l'Amérique portugaise sont indissociables de la réévaluation de la question climatique. L'environnement et les peuples se trouvent imbriqués pour ces hommes de science qui écrivaient sur une réalité tropicale et vivaient eux-mêmes sous les tropiques.

Sans doute la question climatique et géographique concerne également les identités d'autres lieux ou de périodes plus récentes. Avec le recul, on s'aperçoit aujourd'hui que beaucoup d'éléments apparus dans les débats analysés ici ont créé des racines profondes dans l'imaginaire collectif brésilien et constituent déjà des réalités culturelles de la longue durée ${ }^{53}$.

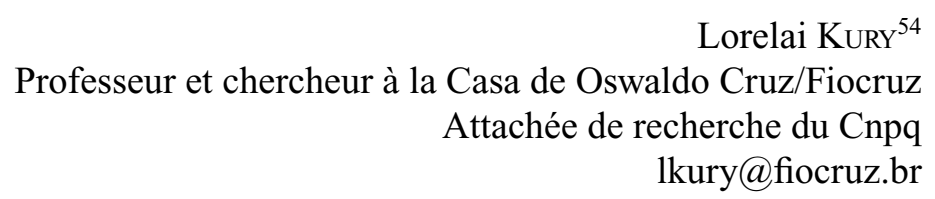

(53) Je remercie Íris Kantor et Joël Deslile pour leurs lectures, ainsi que Marco Morel pour les conversations que nous avons eues sur le thème de cet article.

(54) Texte traduit du portugais par Martim Tavares et Patrice Bret. 